Research Paper

\title{
Distant Metastasis and Survival Outcomes after Computed Tomography-Guided Needle Biopsy in Resected Stage I-III Non-Small Cell Lung Cancer
}

\author{
Yuhui $\mathrm{Xi}^{2 *}$, Jingli Fan ${ }^{1 *}$, Dehai Che ${ }^{1 *}$, Keke Zhai ${ }^{1}$, Tingting Ren ${ }^{1}$, Xiao Feng ${ }^{1}$, Lihua Shang ${ }^{1}$, Jing Hu${ }^{1}$, Yan \\ $\mathrm{Yu}^{1}$, Qingwei Meng ${ }^{1 凶}$ \\ 1. Medical oncology department of Harbin Medical University Cancer Hospital, Harbin, China, 150040; \\ 2. The pathophysiology department of Harbin Medical University, Harbin, China, 150081. \\ * These authors contributed equally to this work. \\ $\triangle$ Corresponding author: Qingwei MENG, Medical oncology department of Harbin Medical University Cancer Hospital, Harbin, China, 150040. Tel: \\ 86-45186298726. Email: mqwei@ems.hrbmu.edu.cn \\ (c) Ivyspring International Publisher. This is an open access article distributed under the terms of the Creative Commons Attribution (CC BY-NC) license \\ (https://creativecommons.org/licenses/by-nc/4.0/). See http://ivyspring.com/terms for full terms and conditions.
}

Received: 2017.04.28; Accepted: 2017.08.31; Published: 2017.09.20

\begin{abstract}
Background and objective Several reports describe the needle-tract implantation after percutaneous needle lung biopsy. The present study evaluated whether preoperative computed tomography-guided needle biopsy (CTNB) affected the distant metastasis and overall survival in patients with early non-small cell lung cancer (NSCLC). Methods A total of 1667 patients with pathological stage I-III NSCLC were assimilated. Of these, 168 patients received preoperative CTNB, whereas 1499 patients were not subjected to any biopsy before surgical resection. Propensity score matching method was adopted to balance the observed covariates between the two groups. Cox regression analysis and Kaplan-Meier estimations were used for survival analysis. Subset analysis was performed in the p-stage $\leq$ II cases. Results The distant metastasis and mortality were not significantly increased for all patients with preoperative CTNB $(P=0.142$ and $P=0.125$, respectively). The subset analysis of $p$-stage $\leq \|$ cases showed that CTNB increased the risk of distant metastasis $(P=0.032)$ while not increasing the risk of mortality $(P=0.086)$. Conclusion CTNB can increase the risk of distant metastasis in the p-stage $\leq$ II patients.
\end{abstract}

Key words: computed tomography-guided needle biopsy; lung cancer; biopsy; distant metastasis; overall survival.

\section{Introduction}

Computed tomography-guided needle biopsy (CTNB) is a well-established and commonly used technique for diagnosing pulmonary nodules with high accuracy and safety [1-5]. Frequent complications associated with CTNB are hemoptysis and pneumothorax [6-8], while majority of them are mild symptoms that rarely need treatment. Fatal complications due to systemic air embolism, hemorrhage, or pericardial tamponade have been reported [7]; however, these are also rare. Needle-tract implantation [9-13] is also a potentially severe complication of CTNB, although it is even rarer.
However, these studies only focused on the short-time complications after preoperative CTNB and seldom mentioned the long-term survival risk.

The purpose of our study was to evaluate whether CTNB is associated with increased risk of both death and distant metastasis by comparing the survival of patients with surgically resected pathological stage I to III non-small cell lung cancer (NSCLC). These patients underwent CTNB before surgical resection at Harbin Medical University Cancer Hospital with a concurrent cohort of patients who did not undergo this procedure. 


\section{Patients and Methods}

\section{Patients}

This retrospective study was approved by Harbin Medical University Cancer Hospital. We identified 2604 patients with NSCLC p-stage I-III who were treated with curative resection between Jan 1, 2011 and Dec 31, 2014, in our hospital. Of these patients in the database, 937 were excluded as they were lost to follow-up $(\mathrm{n}=72)$ or transbronchial biopsy was performed before surgical resection $(\mathrm{n}=$ 865). Thus, 168 patients received CTNB, and 1499 patients did not undergo biopsy before surgical resection on whom the retrospective study was conducted (Fig.1). The patients were staged according to the seventh edition of the American Joint Cancer Committee criteria.

\section{Methods}

Primary outcome variables included distant metastasis-free survival (DMFS) and overall survival (OS). Distant metastasis was defined as any recurrence in pleura, contralateral lung, extrathoracic metastatic disease, or appearance of malignant pleural or pericardial effusions. DMFS was defined as the time from surgery until the first diagnosis of distant metastasis or the last follow-up. Overall survival was defined as the time from surgery to death or last follow-up. Kaplan-Meier method estimated the survival periods.

To compare the differences in the baseline characteristics between CTNB and non-biopsy groups, chi-square tests were used. To balance the observed covariates between CTNB and non-biopsy groups, the propensity score matching method [14] was adopted. Cox regression method was used to analyze the hazard ratios (HRs) and 95\% confidence intervals (CIs) for both mortality and distant metastasis of CTNB group as compared to the non-biopsy group.

Propensity score matching was performed using $\mathrm{R}$ v.2.15, (R Foundation for Statistical Computing, Vienna, Austria). Descriptive analysis, Cox regression, and Kaplan-Meier method were carried out using Statistical Product and Service Solutions (SPSS) v. 22.0. Statistical significance was set at 0.05 , and all tests were two-tailed.

\section{Puncture technique}

Percutaneous transthoracic needle biopsies were performed with CT-guided needle biopsy by radiologists with more than 10 years of experience. Specimens were repeatedly obtained until sufficient material had been collected for pathological examination or until the patient began coughing. The type of biopsy needle was PRECISA 18 GX150MM (HS Hospital Service S.P.A, Italy).

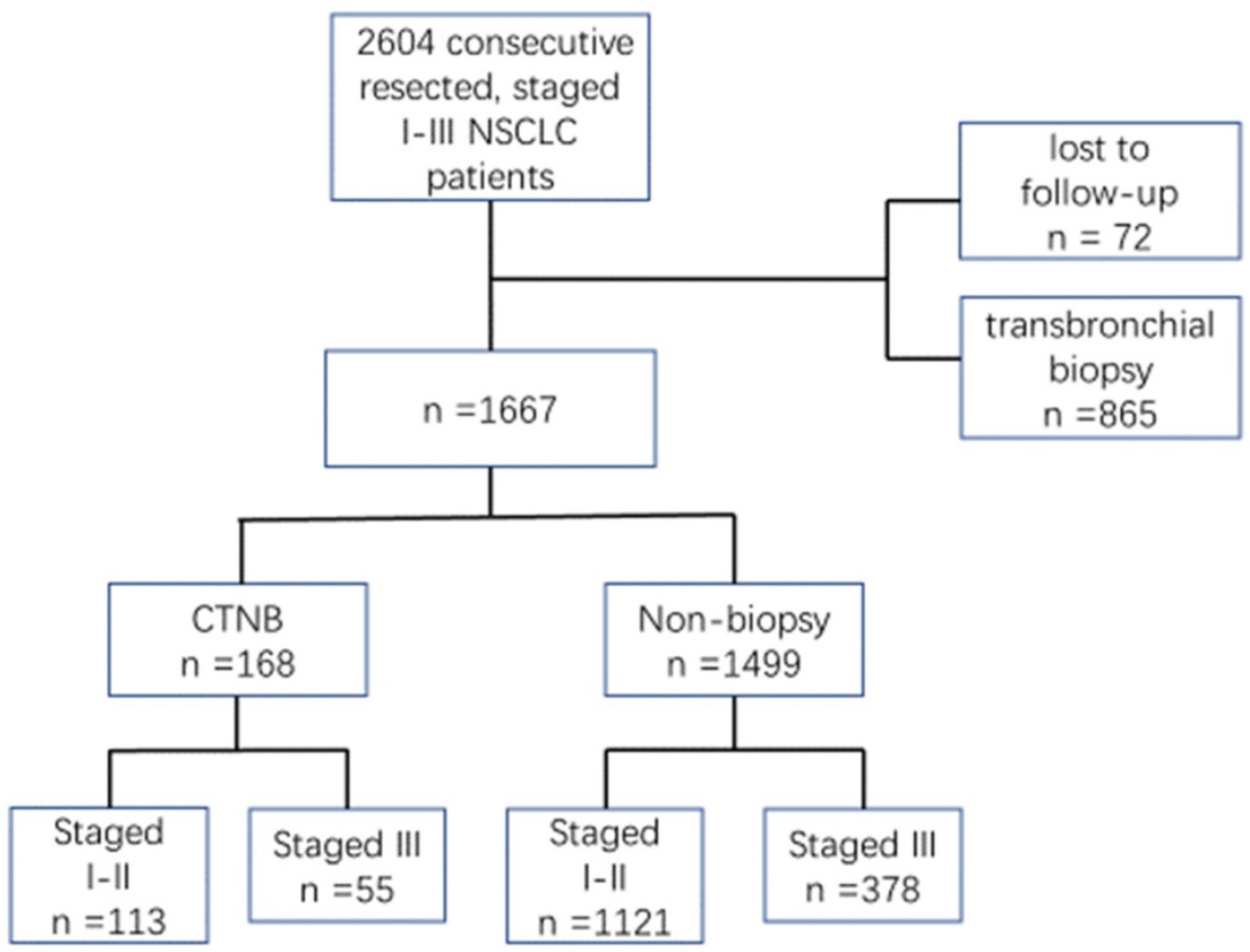

Figure 1. The flow chart of patient selection. NSCLC, non-small cell lung cancer. CTNB, computed tomography-guided needle biopsy. 


\section{Results}

\section{Baseline characteristics of patients at diagnosis}

Of the 1667 patients, 168 (10.08\%) underwent CTNB before surgical resection, and 1499 patients $(89.92 \%)$ were not subjected to any biopsy before surgical resection in the same period. Clinical characteristics of patients are summarized in Table 1. In the entire cohort, no differences were observed between the two groups with respect to age, gender, $\mathrm{N}$ stage, location, as well as, adjuvant radiotherapy. However, CTNB group had poor T stage $(10.72 \%$ vs. $7.20 \%$ stage $\geq \mathrm{T} 3 ; P<0.001)$, poor TNM stage $(32.74 \%$ vs. $25.22 \%$ stage III; $P<0.001$ ), less adenocarcinoma (69.64\% vs. $83.59 \% ; P<0.001)$, and more adjuvant chemotherapy $(51.79 \%$ vs. $31.02 \% ; P<0.001)$ as compared to the non-biopsy group. Propensity score matching was performed on these patients with the ratio 1:1, resulting in 168 patients in both the CTNB and non-biopsy groups, respectively. Matching by propensity score achieved an adequate balance between both groups (Table 1).

When patients were categorized according to
pTNM stages with p-stage $\leq$ II, 113 patients comprised the CTNB group, and 1121 patients formed the non-biopsy group; propensity score matching was also performed in these patients. Variables among the two groups were not statistically significant in the matched patients (Table 2).

\section{Survival curves of DMFS and OS}

DMFS and OS were significantly poorer in the CTNB group than the non-biopsy group $(P=0.006$ and $P=0.005$, respectively; Fig. 2). However, the differences were not statistically significant after matched by propensity score $(P=0.142$ and $P=0.125$, respectively; Fig. 2).

When analyzed in the stage $\leq$ II subgroup, DMFS and OS were also significantly poorer in the CTNB group than the non-biopsy group $(P<0.001$ and $P=$ 0.002, respectively; Fig. 3). DMFS was also significantly poorer in the CTNB group than in the non-biopsy group after matched by the propensity score $(P=0.032$, Fig. 3$)$, whereas a statistically significant difference was not observed in OS among the two groups $(P=0.086$, Fig. 3$)$.

Table 1. Characteristics of patients treated with CTNB vs. non-biopsy in the observational dataset and after propensity score matching for patients with p-stage I-III.

\begin{tabular}{|c|c|c|c|c|c|c|}
\hline \multirow[t]{2}{*}{ Variables } & \multicolumn{3}{|c|}{ Observational dataset $(n=1667)$} & \multicolumn{3}{|c|}{ Propensity score-matched dataset $(n=336)$} \\
\hline & $\begin{array}{l}\text { CTNB } \\
n=168(\%)\end{array}$ & $\begin{array}{l}\text { Non-biopsy } \\
n=1499(\%)\end{array}$ & $\mathrm{p}$ value & $\begin{array}{l}\text { CTNB } \\
\mathrm{n}=168(\%)\end{array}$ & $\begin{array}{l}\text { Non-biopsy } \\
n=168(\%)\end{array}$ & $\mathrm{p}$ value \\
\hline Age & & & 0.858 & & & 0.323 \\
\hline$\geq 60$ & $79(47.02)$ & $694(46.30)$ & & $79(47.02)$ & $70(41.67)$ & \\
\hline$<60$ & $89(52.98)$ & $805(53.70)$ & & $89(52.98)$ & $98(58.33)$ & \\
\hline Gender & & & 0.511 & & & 1.000 \\
\hline Male & $90(53.57)$ & $763(50.90)$ & & $90(53.57)$ & $90(53.57)$ & \\
\hline Female & $78(46.43)$ & $736(49.10)$ & & $78(46.43)$ & $78(46.43)$ & \\
\hline T stage & & & $<0.001^{*}$ & & & 0.989 \\
\hline 1 & $45(26.78)$ & $691(46.10)$ & & $45(26.78)$ & $46(27.38)$ & \\
\hline 2 & $105(62.50)$ & $700(46.70)$ & & $105(62.50)$ & $105(62.50)$ & \\
\hline 3 & $13(7.74)$ & $60(4.00)$ & & $13(7.74)$ & $13(7.74)$ & \\
\hline 4 & $5(2.98)$ & $48(3.20)$ & & $5(2.98)$ & $4(2.38)$ & \\
\hline N stage & & & 0.064 & & & 0.359 \\
\hline 0 & $96(57.14)$ & $990(66.04)$ & & $96(57.14)$ & $102(60.72)$ & \\
\hline 1 & $22(13.10)$ & 169 (11.28) & & $22(13.10)$ & $27(16.07)$ & \\
\hline 2 & $50(29.76)$ & $340(22.68)$ & & $50(29.76)$ & $39(23.21)$ & \\
\hline TNM stage & & & $<0.001^{*}$ & & & 0.145 \\
\hline I & $71(42.26)$ & $890(59.37)$ & & $71(42.26)$ & 89 (52.98) & \\
\hline II & $42(25.00)$ & $231(15.41)$ & & $42(25.00)$ & $34(20.24)$ & \\
\hline III & $55(32.74)$ & $378(25.22)$ & & $55(32.74)$ & $45(26.78)$ & \\
\hline Location & & & 0.825 & & & 0.850 \\
\hline Central & $16(9.52)$ & $135(9.01)$ & & $16(9.52)$ & $15(8.93)$ & \\
\hline Peripheral & $152(90.48)$ & 1364 (90.99) & & $152(90.48)$ & 153 (91.07) & \\
\hline Pathology & & & $<0.001^{*}$ & & & 0.991 \\
\hline Ad & $117(69.64)$ & 1253 (83.59) & & $117(69.64)$ & $116(69.05)$ & \\
\hline Scc & $36(21.43)$ & $184(12.27)$ & & $36(21.43)$ & 37 (22.02) & \\
\hline Others & $15(8.93)$ & $62(4.14)$ & & $15(8.93)$ & $15(8.93)$ & \\
\hline Chemotherapy & & & $<0.001^{*}$ & & & 1.000 \\
\hline Yes & 87 (51.79) & 465 (31.02) & & $87(51.79)$ & 87 (51.79) & \\
\hline No & $81(48.21)$ & $1034(68.98)$ & & $81(48.21)$ & $81(48.21)$ & \\
\hline Radiotherapy ${ }^{\#}$ & & & 0.474 & & & 0.557 \\
\hline Yes & 7 (4.17) & $47(3.14)$ & & 7 (4.17) & $5(2.98)$ & \\
\hline No & $161(95.83)$ & $1452(96.86)$ & & $161(95.83)$ & $163(97.02)$ & \\
\hline
\end{tabular}


Table 2. Characteristics of patients treated with CTNB vs. non-biopsy in the observational data set and after propensity score matching for patients with p-stage $\leq$ II.

\begin{tabular}{|c|c|c|c|c|c|c|}
\hline \multirow[t]{2}{*}{ Clinical factors } & \multicolumn{3}{|c|}{ observational data set $(n=1234)$} & \multicolumn{3}{|c|}{ Propensity score-matched cohort $(n=226)$} \\
\hline & $\begin{array}{l}\text { CTNB } \\
\mathrm{n}=113(\%)\end{array}$ & $\begin{array}{l}\text { non-biopsy } \\
n=1121(\%)\end{array}$ & $\mathrm{p}$ value & $\begin{array}{l}\text { CTNB } \\
\mathrm{n}=113(\%)\end{array}$ & $\begin{array}{l}\text { non-biopsy } \\
n=113(\%)\end{array}$ & $\mathrm{p}$ value \\
\hline Age & & & 0.698 & & & 0.690 \\
\hline$\geq 60$ & $57(50.44)$ & $544(48.53)$ & & $57(50.44)$ & $60(53.10)$ & \\
\hline$<60$ & $56(49.56)$ & $577(51.47)$ & & $56(49.56)$ & $53(46.90)$ & \\
\hline Gender & & & 0.791 & & & 0.894 \\
\hline Male & $58(51.33)$ & $590(52.63)$ & & $58(51.33)$ & $59(52.21)$ & \\
\hline female & $55(48.67)$ & $531(47.37)$ & & $55(48.67)$ & $54(47.79)$ & \\
\hline T stage & & & $<0.001^{*}$ & & & 0.396 \\
\hline 1 & $30(26.55)$ & $586(52.27)$ & & $30(26.55)$ & $27(23.89)$ & \\
\hline 2 & $78(69.03)$ & $502(44.78)$ & & $78(69.03)$ & $76(67.26)$ & \\
\hline 3 & $5(4.42)$ & $33(2.94)$ & & $5(4.42)$ & $10(8.85)$ & \\
\hline $\mathrm{N}$ stage & & & 0.448 & & & 0.857 \\
\hline 0 & $94(83.19)$ & $962(85.82)$ & & $94(83.19)$ & $95(84.07)$ & \\
\hline 1 & $19(16.81)$ & 159(14.18) & & 19(16.81) & 18(15.93) & \\
\hline TNM stage & & & $<0.001^{*}$ & & & 0.087 \\
\hline I & $71(62.83)$ & $890(79.39)$ & & $71(62.83)$ & $83(73.45)$ & \\
\hline II & $42(37.17)$ & $231(20.61)$ & & $42(37.17)$ & $30(26.55)$ & \\
\hline Location & & & 0.319 & & & 0.391 \\
\hline Central & $8(7.08)$ & $112(9.99)$ & & $8(7.08)$ & $5(4.42)$ & \\
\hline Peripheral & 105(92.92) & 1009(90.01) & & $105(92.92)$ & $108(95.58)$ & \\
\hline Pathology & & & $0.001^{*}$ & & & 0.840 \\
\hline Ad & $78(69.03)$ & 919(81.98) & & $78(69.03)$ & $82(72.57)$ & \\
\hline $\mathrm{Scc}$ & $24(21.24)$ & $162(14.45)$ & & $24(21.24)$ & 21(18.59) & \\
\hline Others & $11(9.73)$ & $40(3.57)$ & & $11(9.73)$ & $10(8.85)$ & \\
\hline Chemotherapy\# & & & $<0.001^{*}$ & & & 0.506 \\
\hline Yes & $57(50.44)$ & $275(24.53)$ & & $57(50.44)$ & $52(46.02)$ & \\
\hline No & $56(49.56)$ & $846(75.47)$ & & $56(49.56)$ & $61(53.98)$ & \\
\hline Radiotherapy ${ }^{\#}$ & & & 0.143 & & & 0.313 \\
\hline Yes & $3(2.65)$ & 12(1.07) & & $3(2.65)$ & $1(0.89)$ & \\
\hline No & $110(97.35)$ & 1109(98.93) & & $110(97.35)$ & 112(99.11) & \\
\hline
\end{tabular}

Abbreviations: CTNB, computed tomography-guided needle biopsy; Ad, adenocarcinoma; Scc: squamous-cell carcinoma.

\#: adjuvant therapy. ${ }^{*} P<0.05$.

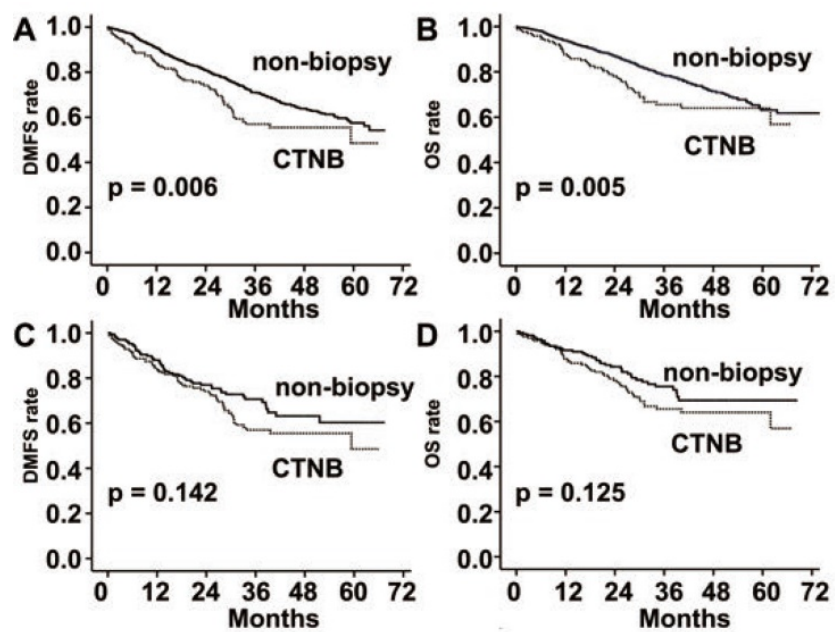

Figure 2. Distant metastasis free survival (DMFS) and overall survival (OS) rates in patients with stage I-III lung cancer. (A) DMFS was significantly reduced in the CTNB group in comparison to the non-CTNB group (CTNB group, $n=168$; non-biopsy group, $n=1499 ; P=0.006$, log-rank test). (B) OS was significantly reduced in the CTNB group as compared to the non-biopsy group (CTNB group, $\mathrm{n}=168$; non-CTNB group, $\mathrm{n}=1499 ; \mathrm{P}=$ 0.005 , log-rank test). (C) DMFS was similar for the two groups in the matched cohort (CTNB group, $\mathrm{n}=168$; non-CTNB group, $\mathrm{n}=168 ; P=0.142$, log-rank test). (D) OS was similar for the two groups in the matched cohort (CTNB group, $\mathrm{n}=168$; non-CTNB group, $\mathrm{n}=168 ; P=0.125$, log-rank test). DMFS: distant metastasis free survival. OS: overall survival.

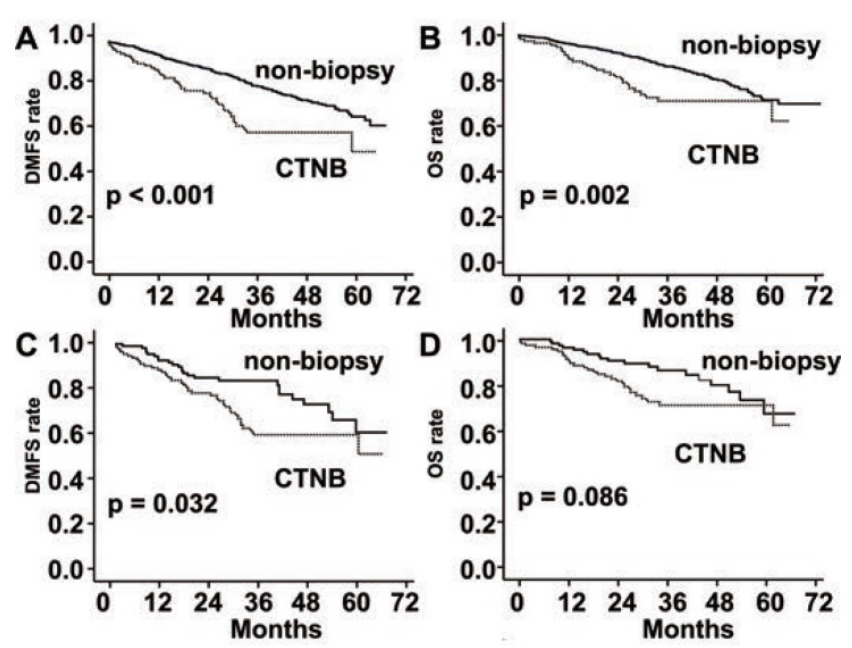

Figure 3. DMFS and OS rates in patients with stage I-II lung cancer. (A) DMFS was significantly reduced in the CTNB group as compared to the non-CTNB group (CTNB group, $n=113$; non-CTNB group, $n=1121 ; P<0.001$, log-rank test). (B) OS was significantly reduced in the CTNB group as compared to the non-CTNB group (CTNB group, $\mathrm{n}=113$; non-CTNB group, $\mathrm{n}=1121 ; P$ $=0.002$, log-rank test). (C) DMFS was significantly reduced in the CTNB group as compared to the non-CTNB group in the matched cohort (CTNB group, $\mathrm{n}=$ 113; non-CTNB group, $\mathrm{n}=113 ; P=0.032$, log-rank test). (D) OS was similar for the two groups in the matched cohort (CTNB group, $n=113$; non-CTNB group, $n=113 ; P=0.086$, log-rank test). DMFS: distant metastasis free survival. OS: overall survival. 
Table 3. Univariable and multivariable analyses of predictors of DMFS and OS for propensity score-matched cohort in p-stage I-III patients

\begin{tabular}{|c|c|c|c|c|c|c|}
\hline \multirow[t]{2}{*}{ Variable } & \multicolumn{3}{|c|}{ Univariable analysis of DMFS } & \multicolumn{3}{|c|}{ Univariable analysis of OS } \\
\hline & HR & $95 \% \mathrm{CI}$ & $\mathrm{p}$ value & HR & $95 \% \mathrm{CI}$ & $\mathrm{p}$ value \\
\hline Age $(\geq 60 v<60)$ & 1.478 & $1.016-2.150$ & $0.041^{* *}$ & 1.569 & $1.031-2.387$ & $0.036^{* *}$ \\
\hline Gender (female $v$ male) & 0.867 & $0.595-1.263$ & 0.458 & 0.709 & $0.464-1.086$ & 0.114 \\
\hline T stage & 1.329 & $1.024-1.726$ & $0.033^{*}$ & 1.280 & $0.952-1.721$ & 0.103 \\
\hline $\mathrm{N}$ stage & 1.636 & $1.334-2.008$ & $<0.001^{* *}$ & 1.754 & $1.395-2.205$ & $<0.001^{* *}$ \\
\hline Location (Peripheral $v$ Central) & 0.675 & $0.391-1.165$ & 0.158 & 0.589 & $0.332-1.044$ & 0.070 \\
\hline \multicolumn{7}{|l|}{ Pathology } \\
\hline $\operatorname{Scc} v$ Ad & 0.558 & $0.314-0.991$ & $0.046^{* *}$ & 0.654 & $0.333-1.283$ & 0.216 \\
\hline Others $v$ Ad & 0.660 & $0.344-1.264$ & 0.210 & 0.886 & $0.421-1.863$ & 0.750 \\
\hline Chemotherapy\# & 0.859 & $0.590-1.250$ & 0.428 & 0.584 & $0.382-0.892$ & $0.013^{* *}$ \\
\hline CTNB or non-biopsy & 1.323 & $0.909-1.927$ & 0.144 & 1.388 & $0.911-2.114$ & 0.127 \\
\hline Radiotherapy $\#$ & 1.231 & $0.502-3.022$ & 0.650 & 1.526 & $0.619-3.764$ & 0.359 \\
\hline
\end{tabular}

Abbreviations: CTNB, computed tomography-guided needle biopsy; Ad, adenocarcinoma; Scc: squamous-cell carcinoma; HR, hazard ratio; CI, confidence interval.

${ }^{*}$ variables were Statistical significance in univariable analysis; ${ }^{* *}$ variables were also Statistical significance in multivariable analysis. \#: adjuvant therapy.

Table 4. Univariable and multivariable analyses of predictors of DMFS and OS for propensity score-matched cohort in p-stage $\leq$ II patients.

\begin{tabular}{|c|c|c|c|c|c|c|}
\hline \multirow[t]{2}{*}{ Clinical factors } & \multicolumn{3}{|c|}{ Univariable analysis of DMFS } & \multicolumn{3}{|c|}{ Univariable analysis of OS } \\
\hline & HR & $95 \% \mathrm{CI}$ & $\mathrm{p}$ value & HR & $95 \% \mathrm{CI}$ & $\mathrm{p}$ value \\
\hline Age $(\geq 60 v<60)$ & 1.057 & $0.646-1.730$ & 0.824 & 1.515 & $0.844-2.718$ & 0.164 \\
\hline Gender (female $v$ male) & 0.815 & $0.497-1.337$ & 0.418 & 0.630 & $0.351-1.132$ & 0.122 \\
\hline T stage & 1.202 & $0.770-1.877$ & 0.419 & 1 & $0.592-1.690$ & 1.000 \\
\hline N stage & 2.387 & $1.380-4.127$ & $0.002^{* *}$ & 2.236 & $1.198-4.175$ & $0.011^{* *}$ \\
\hline $\begin{array}{l}\text { Location (Peripheral } v \text { Central) } \\
\text { pathology }\end{array}$ & 0.645 & $0.259-1.608$ & 0.347 & 0.640 & $0.230-1.781$ & 0.640 \\
\hline $\operatorname{Scc} v$ Ad & 0.363 & $0.191-0.690$ & $0.002^{* *}$ & 0.386 & $0.184-0.812$ & $0.012^{* *}$ \\
\hline Others $v$ Ad & 0.326 & $0.141-0.758$ & $0.009^{* *}$ & 0.390 & $0.150-1.013$ & 0.053 \\
\hline Chemotherapy $\#$ & 1.445 & $0.876-2.384$ & 0.250 & 0.822 & $0.466-1.452$ & 0.500 \\
\hline CTNB or non-biopsy & 1.720 & $1.041-2.844$ & $0.034^{* *}$ & 1.652 & $0.951-2.948$ & 0.090 \\
\hline Radiotherapy\# & 3.829 & $1.194-12.280$ & $0.024^{*}$ & 3.891 & $1.205-12.568$ & $0.023^{*}$ \\
\hline
\end{tabular}

Abbreviations: CTNB, computed tomography-guided needle biopsy; Ad, adenocarcinoma; Scc: squamous-cell carcinoma; HR, hazard ratio; CI, confidence interval.

${ }^{*}$ variables were Statistical significance in univariable analysis; ** variables were also Statistical significance in multivariable analysis. \#: adjuvant therapy.

\section{Univariable and multivariable analysis (relationship between clinical variables and DMFS and OS)}

As is shown in Table 3, during the analysis of all the patients, factors associated with reduced DMFS in the univariable analysis included age $>60$ years, adenocarcinoma, and increasing pathological $\mathrm{T}$ and $\mathrm{N}$ stage. Then, these variables were entered into a backward multivariate analysis and demonstrated that age $>60$ years, adenocarcinoma, and growing pathological $\mathrm{N}$ stage were unfavorable prognostic factors of DMFS. Three clinical variables, including age $>60$ years, poor $\mathrm{N}$ stage, and absence of adjuvant chemotherapy, were associated with reduced OS in the univariable analysis as well as in multivariate analysis (Table 3).

When analyzed in p-stage $\leq$ II subgroup, the factors associated with reduced DMFS in the univariable analysis included CTNB, increasing pathological $\mathrm{N}$ stage, adenocarcinoma and radiotherapy. Then, these variables were entered into a backward multivariate analysis and demonstrated that CTNB, N stage, and adenocarcinoma were unfavorable prognostic factors of DMFS (Table 4).
When examining the predictors of OS, poor N stage, non-radiotherapy, and adenocarcinoma were associated with reduced OS in univariable analysis. When these were analyzed by a backward multivariate analysis, $\mathrm{N}$ stage and adenocarcinoma were found to be independent predictors of OS survival (Table 4).

\section{Discussion}

In our study, the distant metastasis and mortality were not significantly increased after CTNB in p-stage I-III lung cancer patients $(P=0.142$ and $P=$ 0.125 , respectively). The subset analysis of p-stage $\leq$ II cases showed that CTNB increased the risk of distant metastasis $(P=0.032)$ while not increasing the risk of mortality $(P=0.086)$. The difference in mortality caused by CTNB was not statistically significant; the phenomenon that CTNB was inclined to increasing the risk of mortality in p-stage $\leq$ II NSCLC patients, can be noted from our data. A similar study had been performed previously showing that the preoperative transthoracic needle biopsy increased the incidences of pleural recurrences in stage I lung cancer [15-17]. Although these reports have been limited to pleural recurrence after a transthoracic needle biopsy, it still 
indicated that CTNB increases the incidence of distant metastasis in the early stage lung cancer. However, Keisuke et al. speculated that the pleural recurrence was not significantly increased after CTNB in p-stage I lung cancer patients [18]. Keisuke et al. showed that the CTNB group encompassed more pT1a patients than the control group, which might cause the difference.

Moreover, the control group in previous studies not only included patients who did not receive any biopsy but also those who underwent bronchoscopy-based biopsy and other invasive manipulations. In our study, the control group included only patients who did not undergo any biopsy, which eliminated the influence of bronchoscopy and other invasive procedures to the results. We also performed the propensity score matching analysis according to the clinical characteristics of the CTNB and non-biopsy groups to reduce the selection bias.

In summary, we investigated the risk of distant metastasis and mortality after preoperative CTNB in NSCLC patients. The CTNB for suspicious early stage lung cancer should be selected stringently, especially for the patients suspecting to undergo a potentially curative resection. Moreover, imaging findings and diagnosis ought to be taken seriously. Finally, other diagnosis methods, such as the detection of cancer driver gene in blood serum should also be focused upon for further studies.

\section{Abbreviations}

CTNB: computed tomography-guided needle biopsy; NSCLC: non-small cell lung cancer; DMFS: distant metastasis-free survival; OS: overall survival

\section{Acknowledgments}

This study was supported by grants from the National Natural Science Foundation of China [grant numbers 81672931, 81100191, 81501960, 81602717, 81673024]; China postdoctoral science foundation grant [grant number 2016M591552]; Heilongjiang postdoctoral fund [grant number LBH-Z16254]. Thanks to MedSci (Shanghai, China) for providing language help.

\section{Informed consent}

Informed consent was obtained from all individual participants included in the study.

\section{Competing Interests}

The authors have declared that no competing interest exists.

\section{References}

1. Hiraki T, Mimura H, Gobara H, Iguchi T, Fujiwara H, Sakurai J, et al. CT fluoroscopy-guided biopsy of 1,000 pulmonary lesions performed with 20-gauge coaxial cutting needles: diagnostic yield and risk factors for diagnostic failure. Chest. 2009; 136: 1612-7.

2. Lee SM, Park CM, Lee KH, Bahn YE, Kim JI, Goo JM. C-arm cone-beam CT-guided percutaneous transthoracic needle biopsy of lung nodules: clinical experience in 1108 patients. Radiology. 2014; 271: 291-300.

3. DiBardino DM, Yarmus LB, Semaan RW. Transthoracic needle biopsy of the lung. Journal of thoracic disease. 2015; 7: S304-16.

4. Li Y, Du Y, Yang HF, Yu JH, Xu XX. CT-guided percutaneous core needle biopsy for small $(</=20 \mathrm{~mm})$ pulmonary lesions. Clinical radiology. 2013; 68: e43-8

5. Yang W, Sun W, Li Q, Yao Y, Lv T, Zeng J, et al. Diagnostic Accuracy of CT-Guided Transthoracic Needle Biopsy for Solitary Pulmonary Nodules. PloS one. 2015; 10: e0131373.

6. Nour-Eldin NE, Alsubhi M, Emam A, Lehnert T, Beeres M, Jacobi V, et al. Pneumothorax Complicating Coaxial and Non-coaxial CT-Guided Lung Biopsy: Comparative Analysis of Determining Risk Factors and Management of Pneumothorax in a Retrospective Review of 650 Patients. Cardiovascular and interventional radiology. 2016; 39: 261-70.

7. Tomiyama N, Yasuhara Y, Nakajima Y, Adachi S, Arai Y, Kusumoto M, et al. CT-guided needle biopsy of lung lesions: a survey of severe complication based on 9783 biopsies in Japan. European journal of radiology. 2006; 59: 60-4.

8. Wang Y, Jiang F, Tan X, Tian P. CT-guided percutaneous transthoracic needle biopsy for paramediastinal and nonparamediastinal lung lesions: Diagnostic yield and complications in 1484 patients. Medicine. 2016; 95: e4460.

9. Kara M, Alver G, Sak SD, Kavukcu S. Implantation metastasis caused by fine needle aspiration biopsy following curative resection of stage IB non-small cell lung cancer. European journal of cardio-thoracic surgery: official journal of the European Association for Cardio-thoracic Surgery. 2001; 20: 868-70.

10. McDonald CF, Baird L. Risk of needle track metastasis after fine needle lung aspiration in lung cancer--a case report. Respiratory medicine. 1994; 88: 631-2

11. Voravud N, Shin DM, Dekmezian RH, Dimery I, Lee JS, Hong WK. Implantation metastasis of carcinoma after percutaneous fine-needle aspiration biopsy. Chest. 1992; 102: 313-5.

12. Wolinsky $\mathrm{H}$, Lischner MW. Needle track implantation of tumor after percutaneous lung biopsy. Annals of internal medicine. 1969; 71: 359-62.

13. Yoshikawa T, Yoshida J, Nishimura M, Yokose T, Nishiwaki Y, Nagai K. Lung cancer implantation in the chest wall following percutaneous fine needle aspiration biopsy. Japanese journal of clinical oncology. 2000; 30: 450-2.

14. Stuart EA, Lee BK, Leacy FP. Prognostic score-based balance measures can be a useful diagnostic for propensity score methods in comparative effectiveness research. Journal of clinical epidemiology. 2013; 66: S84-S90.

15. Inoue M, Honda O, Tomiyama N, Minami M, Sawabata N, Kadota $\mathrm{Y}$, et al. Risk of pleural recurrence after computed tomographic-guided percutaneous needle biopsy in stage I lung cancer patients. The Annals of thoracic surgery. 2011; 91: 1066-71.

16. Kashiwabara K, Semba H, Fujii S, Tsumura S. Preoperative Percutaneous Transthoracic Needle Biopsy Increased the Risk of Pleural Recurrence in Pathological Stage I Lung Cancer Patients with Sub-pleural Pure Solid Nodules. Cancer investigation. 2016; 34: 373-7.

17. Wisnivesky JP, Henschke CI, Yankelevitz DF. Diagnostic percutaneous transthoracic needle biopsy does not affect survival in stage I lung cancer. American journal of respiratory and critical care medicine. 2006; 174: 684-8.

18. Asakura K, Izumi Y, Yamauchi Y, Nakatsuka S, Inoue M, Yashiro H, et al. Incidence of pleural recurrence after computed tomography-guided needle biopsy in stage I lung cancer. PloS one. 2012; 7: e42043. 